\title{
DOES THE RADIATIVE AVALANCHE FUELING WORK IN ANY ACTIVE GALACTIC NUCLEI?
}

\author{
Yoshiaki Taniguchi \\ Astronomical Institute, Tohoku University, Aoba, Sendai 980-77, Japan
}

\begin{abstract}
Recently Umemura, Fukue, \& Mineshige (1997) proposed the radiative avalanche fueling to active galactic nuclei; gas accretion is driven by radiation drag exerted by stellar radiation from circumnuclear starburst regions. This mechanism is also interesting in terms of starburst-AGN connections. We therefore present observational tests for the radiative avalanche fueling. Our tests, however, show that gas accretion rates driven by the radiative avalanche are significantly lower than those expected from the standard accretion theory applied for typical active galactic nuclei with the circumnuclear starburst regions. Instead we propose an alternative, possible starburst-AGN connection; a minor merger with a nucleated satellite drives circumnuclear starbursts and then leads to gas fueling onto the central engine as the merger proceeds.
\end{abstract}

Subject headings: accretion - galaxies: active - galaxies: nuclei - quasars: general

Accepted for publications in THE ASTROPHYSICAL JOURNAL (LETTERS) 1997 July

\section{INTRODUCTION}

Since the starburst activity is observed in the circumnuclear regions of some active galactic nuclei (AGNs), possible starburst-AGN connections have been discussed for these two decades (Weedman 1983; Terlevich \& Melnick 1985; Norman \& Scoville 1988; Sanders et al. 1988; Heckman et al. 1989; Rieke 1992; Scoville 1992; Taniguchi 1987, 1992; 
Mouri \& Taniguchi 1992; Terlevich et al. 1992). The discussion has been made in terms of; a) formation of compact nuclei (e.g., Weedman 1983), b) AGN modeling without a supermassive black hole (e.g., Terlevich et al. 1992), or c) gas fueling triggered by the circumnuclear starbursts (e.g., Norman \& Scoville 1988; Taniguchi 1992).

Recently, Umemura, Fukue, \& Mineshige (1997a, 1997b; hereafter UFM97) proposed a novel idea that mass accretion onto a supermassive black hole may be triggered by radiation drag exerted by stellar radiation from the circumnuclear starburst regions (the radiative avalanche fueling), based on the well-known physical process, so-called Poynting-Robertson effect. Since it is known that a number of AGNs have actually luminous circumnuclear starburst (hereafter CNSB) regions (e.g., Telesco et al. 1984; Heckman et al. 1986; Keel 1987; Wilson 1988; Storchi-Bergmann et al. 1996a, 1996b and references therein), it is worth testing if this new mechanism works from an observational point of view.

\section{A BRIEF SUMMARY OF THE RADIATIVE AVALANCHE FUELING}

We give a brief summary of the radiative avalanche fueling proposed by UFM97. The radiative avalanche means that gas accretion is driven by radiation drag exerted by stellar radiation from circumnuclear starburst regions. If a surface layer of a rotating nuclear gas disk is irradiated by the massive stars in the CNSB regions, the gas on the surface could lose angular momentum via radiation drag (i.e., Poynting-Robertson effect; Poynting 1903; Robertson 1937), giving rise to an avalanche of the gas layer.

If a CNSB region with a total luminosity of $L_{\mathrm{CNSB}}$ is located at a radius $R$ with an effective size $\Delta R$ (i.e., the effective diameter of a star-forming clump), the accretion rate due to the avalanche at a radius $r(<R)$ is given by

$$
\dot{M}_{\mathrm{rad}} \simeq 0.2 \eta_{\mathrm{r}} \sin \theta_{d}\left(\frac{r}{R}\right)^{2}\left(\frac{L_{\mathrm{CNSB}}}{3 \times 10^{12} L_{\odot}}\right) \quad M_{\odot} \mathrm{y}^{-1}
$$

where $\eta_{\mathrm{r}}$ is the efficiency of radiative accretion $(\sim 0.1-1)$, and $\left(\sin \theta_{d}\right) \times(r / R)^{2}$ is the irradiation efficiency onto the surface layer at radius $r$ where $\sin \theta_{d} \sim \Delta R /(R-r)$. In the following discussion, we adopt $\eta_{\mathrm{r}}=1$. In UFM97, the factor of $\sin \theta_{d}$ is not seriously

taken into account. However, the recent Hubble Space Telescope observations have shown that the CNSB regions consist of a number of star-forming clumps and each clump (i.e., a star cluster) has an effective diameter of $\sim 10 \mathrm{pc}$ at most (Barth et al. 1995; Maoz et al. 1996). This compact nature is also observed in the archetypical giant HII region, 30 Dor in LMC (Weigelt et al. 1991). Given the typical radius of $1 \mathrm{kpc}$ for the CNSB 
rings (Storchi-Bergman et al. 1996a, 1996b), we estimate $\sin \theta_{d} \sim 0.01$. Furthermore, the geometrical dilution factor is estimated to be $(r / R)^{2} \sim 0.01$ for $r=100 \mathrm{pc}$ and $R=1 \mathrm{kpc}$. Therefore, the accretion rate driven by the radiative avalanche is much lower than that estimated in UFM97.

The characteristic accretion timescale for the radiative avalanche is estimated as

$$
t_{\mathrm{rad}} \simeq 2.4 \times 10^{6}\left(\frac{L_{\mathrm{CNSB}}}{3 \times 10^{12} L_{\odot}}\right)^{-1}\left(\frac{R}{100 \mathrm{pc}}\right)^{2}\left(\frac{f_{\mathrm{dg}}}{10^{-2}}\right)^{-1}\left(\frac{a_{\mathrm{d}}}{0.1 \mu \mathrm{m}}\right)\left(\frac{\rho_{\mathrm{s}}}{\mathrm{g} \mathrm{cm}^{-3}}\right) \mathrm{yr}
$$

where $f_{\mathrm{dg}}$ is the dust-to-gas mass ratio, $a_{\mathrm{d}}$ is the size of dust grains, and $\rho_{\mathrm{s}}$ is the mass density of solid material within the grain. The CNSBs in AGNs are usually observed as ringed (or armed) star-forming clumps and their typical radii range from several $100 \mathrm{pc}$ to 1 kpc (Telesco et al. 1984; Boer \& Schulz 1993; Genzel et al. 1995; Storchi-Bergmann, Wilson, \& Baldwin 1996; Storchi-Bergmann et al. 1996b; Maoz et al. 1996). The luminosities of the CNSBs amount to $\sim 10^{11} L_{\odot}$ in both NGC 1068 (e.g., Telesco et al. 1984) and NGC 7469 (e.g., Genzel et al. 1995). If we adopt both $L_{\mathrm{CNSB}}=10^{11} L_{\odot}$ and $R=1 \mathrm{kpc}$, we obtain $t_{\text {rad }} \sim 7 \times 10^{9}$ years. This timescale seems too long to cause efficient fueling to AGN although it is known that the ages of the CNSBs in Seyfert galaxies are estimated to be $\sim 10^{8}$ years, being older significantly than those of on-going starbursts, $\sim 10^{7}$ years (Glass \& Moorwood 1985; Mouri \& Taniguchi 1992; Taniguchi \& Mouri 1992; Dultzin-Hacyan \& Benitez 1994; Oliva et al. 1995; Hunt et al. 1997).

\section{OBSERVATIONAL TESTS FOR THE RADIATIVE AVALANCHE FUELING}

\subsection{Circumnuclear Starbursts around Seyfert Nuclei}

Prior to the observational tests for the radiative avalanche fueling, we argue the occurrence of the CNSBs around Seyfert nuclei. Based on the IRAS data base, it has been discussed that Seyfert 2 nuclei (hereafter S2s) tend to show excess mid- (MIR) and far-infrared (FIR) emission with resect to Seyfert 1s (S1s) (Rodríguez-Espinosa, Rudy, \& Jones 1987; Dahari \& DeRobertis 1988; Heckman et al. 1989; Maiolino et al. 1995). Also, molecular gas content is higher in S2s than in S1s (Heckman et al. 1989). These observations are usually interpreted as that S2s tend to have luminous CNSB regions more frequently than S1s. However, if there would be some extended dusty clouds around Seyfert nuclei, they show excess emission at FIR because the equilibrium temperature is as cold as 
100 K (Pier \& Krolik 1993; Granato, Danese, \& Francheschini 1996; Taniguchi et al. 1997). This means that S2s may have brighter FIR emission if they would have more extended dusty regions around their nuclei. Thus we cannot conclude that almost all S2s have the CNSB solely from the analysis of FIR data.

Pogge (1989) made a narrow-band emission-line imaging survey of 20 nearby Seyfert galaxies and found that the CNSB regions are present only in $\sim 30$ percent of the S2s while no CNSB is found in the S1s. Although there is a famous S1 with the intense CNSB, NGC 7469 (Heckman et al. 1986; Wilson et al. 1986, 1991; Keto et al. 1992; Mauder et al. 1994; Genzel et al. 1995), it is known that there is a few S1s with CNSBs (Oliva et al. 1995; Hunt et al. 1997). Hunt et al. (1997) showed form their NIR multi-color imaging study that there is little evidence for CNSBs in S1s and even if CNSB events would occur in them, they occurred more than $10^{9}$ years ago. Thus we consider that the radiative avalanche triggered by the CNSBs cannot work in most S1s in the nearby universe because of the absence of CNSBs in them. Therefore, possible candidates of Seyfert nuclei triggered by the radiative avalanche may be about one third of S2s and a few S1s which have the luminous CNSBs around the nuclei. In the following subsections, we present observational tests for typical Seyfert and LINER円 nuclei with CNSBs. Further we mention about the case of ultraluminous infrared galaxies (Sanders et al. 1988).

\section{2. $\quad$ Seyfert Nuclei with Luminous Circumnuclear Starbursts}

Among the Seyfert nuclei with CNSBs (Storchi-Bergmann et al. 1996a, 1996b and references therein), we study the two archetypical Seyfert nuclei, NGC 1068 (S2) and NGC 7469 (S1). In order to examine if gas accretion driven by the radiative avalanche is high enough to achieve the observed bolometric luminosities of central engines, we compare the two accretion rates; 1 ) the gas accretion rate driven by radiative avalanche due to the stellar lights form the CNSBs [see equation (1)], and 2) the gas accretion rate estimated with the standard accretion theory (Rees 1984),

$$
\dot{M}_{\mathrm{acc}}=\frac{L_{\mathrm{bol}}}{\eta_{\mathrm{acc}} c^{2}} \simeq 0.02\left(\frac{L_{\mathrm{bol}}}{10^{44} \mathrm{erg} \mathrm{s}^{-1}}\right)\left(\frac{\eta_{\mathrm{acc}}}{0.1}\right)^{-1} M_{\odot} \mathrm{y}^{-1}
$$

where $\eta_{\text {acc }}$ is the conversion efficiency from the gravitational energy to the radiation. As for AGNs with CNSBs, UFM97 considered that only high-energy photons come from the

\footnotetext{
${ }^{1}$ LINER $=$ Low Ionization Nuclear Emission-line Region (Heckman 1980).
} 
central engine while the other radiation (e.g., from FIR to UV) comes form the CNSBs. Thus they used the X-ray luminosity instead $L_{\text {bol }}$ when they estimated $\dot{M}_{\text {acc }}$. However, this assumption seems inadequate. The reason for this is, for example, that much amount of infrared emission of such galaxies comes from the dusty tori (Pier \& Krolik 1993; Pier et al. 1994; see for the extreme case, Taniguchi et al. 1997). Therefore, in the later discussion, we use the $L_{\text {bol }}$ derived from the spatially-resolved observations in the estimates of $\dot{M}_{\text {acc }}$. Also, we use the CNSB luminosities based on the spatially-resolved observations in the estimates of $\dot{M}_{\text {rad }}$. Our method makes it possible to perform accurate tests.

NGC 1068 is one of the most luminous Seyfert galaxies in the local universe. Its bolometric luminosity of the central engine is estimated to be $L_{\mathrm{bol}} \simeq 8.5 \times 10^{44}\left(f_{\mathrm{refl}} / 0.01\right)^{-1}(D / 22 \mathrm{Mpc})^{2} \mathrm{erg} \mathrm{s}^{-1}$ where $f_{\text {refl }}$ is the fraction of nuclear flux reflected into our line of sight and $D$ is the distance to NGC 1068 (Pier et al. 1994). Adopting the fiducial values in Pier et al. (1994), we obtain the gas accretion rate, $\dot{M}_{\text {acc }} \simeq 0.17 M_{\odot} \mathrm{y}^{-1}$. Next we estimate the accretion rate driven by radiative avalanche. The bolometric luminosity of the CNSB regions is estimated to be $L_{\mathrm{CNSB}} \simeq 2.2 \times 10^{11} L_{\odot}$ at distance of $22 \mathrm{Mpc}$ (Telesco et al. 1984). The radius of the CNSB ring is about $1 \mathrm{kpc}$ (Telesco et al. 1984; Baldwin et al. 1987)]. Although there is no direct measurement of the vertical width $(\Delta R)$ of the star-forming clumps, it is estimated to be $10 \mathrm{pc}$ at most (Weigelt et al. 1991; Barth et al. 1995; Maoz et al. 1996). If we consider the radiative avalanche at $r=100 \mathrm{pc}$, the irradiation efficiency is $\sin \theta_{d}(r / R)^{2} \simeq \Delta R r^{2} /\left[(R-r) R^{2}\right] \sim 1.1 \times 10^{-4}$. We thus obtain the accretion rate by radiative avalanche, $\dot{M}_{\text {rad }} \simeq 1.6 \times 10^{-6} M_{\odot} \mathrm{y}^{-1}$ for $r=100$ pc. Since this accretion rate is much smaller than that estimated from the accretion theory, we consider that the radiative avalanche does not work in NGC 1068.

NGC 7469 is also one of the famous Seyfert galaxies with the luminous CNSB regions (Cutri et al. 1984; Heckman et al. 1986; Wilson et al. 1986, 1991; Miles, Houck, \& Hayward 1994; Mauder et al. 1994; Genzel et al. 1995). The bolometric luminosity of the central engine including the stellar luminosity of the host galaxy is $8.9 \times 10^{44} \mathrm{erg} \mathrm{s}^{-1}$ at a distance of $98 \mathrm{Mpc}$ (Genzel et al. 1995). Since the contribution of the central engine to this luminosity is $\sim 40$ percent (Kotilainen et al. 1992), we obtain $L_{\mathrm{bol}} \simeq 3.5 \times 10^{44} \mathrm{erg} \mathrm{s}^{-1}$ and thus the required gas accretion rate is $\dot{M}_{\text {acc }} \simeq 0.07 M_{\odot} \mathrm{y}^{-1}$. On the other hand, the CNSB ring has the luminosity of $4.5 \times 10^{11} L_{\odot}$ at a radius of $720 \mathrm{pc}$. Assuming $\Delta R=10 \mathrm{pc}$ again, we obtain $\dot{M}_{\text {rad }} \simeq 9.3 \times 10^{-6} M_{\odot} \mathrm{y}^{-1}$ for $r=100 \mathrm{pc}$, being much lower than the expected

\footnotetext{
${ }^{2}$ Braatz et al. (1993) and Cameron et al. (1993) detected the inner MIR component with size of $\sim 100 \mathrm{pc}$. Since, however, the dusty torus in NGC 1068 is considered to be extended at radius of 100 pc (Pier \& Krolik 1993), this MIR component may not be star-forming regions. Even if this component is the star-forming regions, the accretion rate by radiative avalanche is still smaller by one order than the predicted value.
} 
value. Accordingly, we have shown that the contribution of the gas accretion driven by radiative avalanche is negligibly small in both the Seyfert nuclei, NGC 1068 and NGC 7469.

\section{3. $\quad$ LINERs with Circumnuclear Starbursts}

It is also known that some LINERs have CNSBs (Storchi-Bergmann et al. 1996a, 1996b and references therein). Here we investigate a case of NGC 1097 (Keel 1983; Hummel, van der Hulst, \& Keel 1987; Storchi-Bergmann, Baldwin, \& Wilson 1993; Storchi-Bergmann et al. 1995; Barth et al. 1995). Although this galaxy was originally classified as a LINER (Keel 1983), the double-peaked broad line emission appeared since 1991 (Storchi-Bergmann et al. 1993, 1995). In terms of the standard model, some sporadic accretion events would occur since 1991 (Eracleous et al. 1995). It is interesting to examine if this accretion is due to the radiative avalanche. Although there is no measurement of the bolometric luminosity of the central engine, using the observed $\mathrm{H} \alpha$ luminosity of the broad line component, $L(\mathrm{H} \alpha)=5.5 \times 10^{39} \mathrm{erg} \mathrm{s}^{-1}$ at a distance of $14.5 \mathrm{Mpc}$ (Storchi-Bergmann et al. 1993) we may estimate $L_{\text {bol }} \sim 100 L(\mathrm{H} \alpha) \simeq 5.5 \times 10^{41} \mathrm{erg} \mathrm{s}^{-1}$ (Ward et al. 1987; H. Mouri, private communication). Thus we obtain the gas accretion rate, $\dot{M}_{\text {acc }} \simeq 1.1 \times 10^{-4} M_{\odot}$ $\mathrm{y}^{-1}$. Adopting that the semi-minor radius of the CNSB ring is $650 \mathrm{pc}$, the width of the ring is 5 pc (Barth et al. 1995), and $L_{\mathrm{CNSB}}=4.9 \times 10^{7} L_{\odot}$ (Hummel et al. 1987), we obtain $\dot{M}_{\text {rad }} \simeq 1.4 \times 10^{-9} M_{\odot} \mathrm{y}^{-1}$ for $r=100$ pc. Since this rate is much lower than the expected value, we conclude that the radiative avalanche does not work in NGC 1097.

\subsection{Ultraluminous Infrared Galaxies}

Our final test is applied to the ultraluminous infrared galaxies (ULIGs; Sanders et al. 1988). Because of both their huge bolometric luminosities of the starbursts, $L_{\mathrm{CNSB}} \sim 10^{12} L_{\odot}$ and the compact nature of the starbursts, $R \sim 50$ pc (Condon et al. 1991), the radiative avalanche would work most efficiently in the ULIGs. Since it is likely that the ULIG nuclei are surrounded by the CNSB regions (i.e., the geometrical dilution factor $\sim 1$ ), we may obtain $\dot{M}_{\text {rad }} \simeq 0.07 M_{\odot} \mathrm{y}^{-1}$. It is here noted that the circumnuclear molecular gas mass in ULIGs is typically $\sim 10^{10} M_{\odot}$ (Scoville et al. 1991). If this gas would be supplied to the nuclear region within a merger timescale of $\sim 10^{9}$ years, the average gas accretion rate amounts to $\sim 10 M_{\odot} \mathrm{y}^{-1}$. In fact, many numerical simulations have shown that major mergers between two gas-rich galaxies can supply a lot of gas within a reasonable timescale (Mihos \& Hernquist 1994b and references therein). Since this rate is much higher than that by the radiative avalanche, we consider that the dynamical accretion driven by the merger 
is the dominant fueling mechanism rather than the radiative avalanche.

\section{DISCUSSION: AN ALTERNATIVE STARBURST-AGN CONNECTION}

Although we have shown that the radiative-avalanche fueling may not work in the actual AGNs, it is still worth discussing possible starburst-AGN connections because a non-negligible number of AGNs have indeed the CNSBs. We therefore consider another possible connection in this section.

Given the standard scenario for AGNs, the gas fueling is one of the most important physical processes for triggering AGNs (Shlosman, Begelman, \& Frank 1990). As far as Seyfert nuclei are concerned ${ }^{\natural}$, possible fueling mechanisms may be either tidal triggering by a companion galaxy (Noguchi 1988; see for a review Barnes \& Hernquist 1992), or minor merger with a satellite galaxy (Gaskell 1985; Mihos \& Hernquist 1994a; Hernquist \& Mihos 1995). Although Seyfert galaxies tend to have their companion galaxies, the percentage of Seyfert galaxies with physical companions is 12 percent at most (Rafanelli, Violato, \& Baruffolo 1995 and references therein). Further, there is no preferred kind of interaction (prograde, polar, or retrograde) among the Seyfert galaxies with physical companions (Keel 1996) although the efficient fueling would occur in prograde interacting systems. Therefore, the majority of Seyfert galaxies have no relation with tidal interaction and thus should be triggered by certain internal mechanisms. On the other hand, since most galaxies have their satellite galaxies (Zaritsky et al. 1997 and references therein), it is likely that they have already experienced some minor mergers during their lives (Ostriker \& Tremaine 1975; Tremaine 1981). Hence it is suggested strongly that the minor merger hypothesis has the great advantage rather than the tidal triggering.

Here we propose a new possible starburst-AGN connection based on the minor merger hypothesis. Recently, Taniguchi \& Wada (1996) argued that a minor merger with a nucleated satellite causes the efficient gas fueling, leading both to circumnuclear starbursts and then to nuclear starbursts, because of the dynamical disturbance driven by a supermassive binary (i.e., the host nucleus and the satellite one) during the course of the minor merger. If a host galaxy disk has abundant gas, a circumnuclear gas disk would be formed prior to the attack by the satellite nucleus (Hernquist \& Mihos 1995). Therefore, if this is the case, circumnuclear starburst would occur in an early stage of the

\footnotetext{
${ }^{3}$ Ho, Filippenko, \& Sargent (1997) show that bars have a negligible effect on the strength of AGNs in their sample of over 300 spiral galaxies. Therefore, we do not consider that the gas fueling driven by bars is important.
} 
supermassive binary formation. As the separation between the nuclei decreases, the gas clouds are channeled gradually into the host nucleus during the course of merger evolution. The merger timescale from a radius of $\sim 1 \mathrm{kpc}$ to the nuclear region may be of order $10^{8}$ years (Taniguchi \& Wada 1996). Accordingly, we are able to explain the simultaneous presence of both older CNSB regions and the fueled AGNs; i.e., Seyfert nuclei with CNSBs (the majority may be S2s). On the other hand, if a host galaxy disk has little gas, no CNSB would occur but the central engine would be fueled finally because any disk galaxies may have a bit of nuclear gasf (e.g., Taniguchi et al. 1994), giving rise to S1s with no CNSB. Therefore, a variety of Seyfert nuclei can arise as due to different gaseous contents in the hosts.

It seems hard to detect direct evidence for minor mergers in some cases because the dynamical perturbation should be smaller significantly than that of typical galaxy interaction. The long timescale of the merger, $\sim 10^{9}$ years, may lead to the smearing of the relic of minor mergers. Thus some well-evolved minor mergers may be observed as ordinary-looking isolated galaxies. However, it is known that minor mergers cause the kinematic heating of host disks (Quinn, Hernquist, \& Fullagar 1993). Such disk galaxies may be classified as S0 or amorphous galaxies which are frequently observed in the Seyfert hosts (Simkin, Su, \& Schwarz 1980; MacKenty 1990). Therefore, the minor merger hypothesis can also explain the observed diversity of the morphological properties of Seyfert hosts (Simkin et al. 1980; Arsenault 1989; MacKenty 1990; Moles, Márquez, \& Pérez 1995). It is also interesting to note that minor merger hypothesis can also be responsible for the observed random orientation of radio jets in Seyfert nuclei with respect to the host disk axis as discussed by Schmitt et al. (1997 and references therein). Finally, we mention that the merger scenario is also applicable to the more luminous starburst-AGN (i.e., ULIG-quasar) connection provided that major mergers between or among nucleated gas-rich galaxies are progenitors of quasars (Sanders et al. 1988).

We would like to thank Masayuki Umemura, Jun Fukue, Shin Mineshige, Keiichi Wada, Toru Yamada, Hideaki Mouri, and Neil Trentham for useful discussion and comments. This work was financially supported in part by Grant-in-Aids for the Scientific Research (No. 0704405) of the Japanese Ministry of Education, Culture, Sport, and Science.

\footnotetext{
${ }^{4}$ Typical Seyfert nuclei need gas of $\sim 10^{6} M_{\odot}$ as the fuel to sustain the central engine if the accretion rate is $\sim 0.01 M_{\odot} \mathrm{y}^{-1}$ and the duration of active phase is $\sim 10^{8}$ years.
} 


\section{REFERENCES}

Arsenault, R. 1989, A \& A, 217, 66

Baldwin, J. A., Wilson, A. S., \& Whittle, M. 1987, ApJ, 319, 84

Barnes, J. E., \& Hernquist, L. E. 1992, ARA \& A, 30, 705

Barth, A. J., Ho, L. C., Filippenko, A. V., \& Sargent, W. L. W. 1995, AJ, 110, 1009

Boer, B., \& Schulz, H. 1993, A \& A, 277, 397

Braatz, J. A., Wilson, A. S., Gezari, D. Y., Varosi, F., \& Beichman, C. A. 1993, ApJ, 409, L5

Cameron, M., Storey, J. W. V., Rotaciuc, V., Genzel, R., Verstrate, L., Drapatz, S.,

Siebenmorgen, R., \& Lee, T. J. 1993, ApJ, 419, 136

Condon, J. J., Huang, Z. -P., Yin, Q. F., \& Thuan, T. X. 1991, ApJ, 378, 65

Cutri, R. M., Rudy, R. J., Rieke, G. H., Tokunaga, A., \& Willner, S. P. 1984, ApJ, 280, 521

Dahari, O., \& DeRobertis, M. M. 1988, ApJS, 67, 249

Dultzin-Hacyan, D., \& Benitez, E. 1994, A \& A, 291, 720

Eracleous, M., Livio, M., Halpern, J. P., \& Storchi-Bergmann, T. 1995, ApJ, 438, 610

Gaskell, C. M. 1985, Nature, 315, 386

Glass, I. S., \& Moorwood, A. F. M. 1985, MNRAS, 214, 429

Granato, G. L., Danese, L., \& Francheschini, A. 1996, ApJ, 460, L11

Heckman, T. M. 1980, A \& A, 87, 152

Heckman, T. M., Beckwith, S., Blitz, L., Skrutskie, M., \& Wilson, A. S. 1986, ApJ, 305, 157

Heckman, T. M., Blitz, L., Wilson, A. S., Armus, L., \& Miley, G. K. 1989, ApJ, 342, 735

Hernquist, L., \& Mihos, C. J. 1995, ApJ, 448, 41

Ho, L. C., Filippenko, A. V., \& Sargent, W. L. W. 1997, ApJ, in press

Hummel, E., van der Hulst, J. M., \& Keel, W. C. 1987, A \& A, 172, 32

Hunt, L. K., Malkan, M. A., Salvati, M., Mandolesi, N., Palazzi, E., \& Wade, R. 1997, ApJS, 108, 229

Keto, E., Ball, R., Arens, J., Jernigan, G., \& Mixner, M. 1992, ApJ, 389, 223

Keel, W. C. 1983, ApJ, 269, 466

Keel, W. C. 1987, in Star Formation in Galaxies, ed. C. J. Lonsdale (NASA CP-2466), 661

Keel, W. C. 1996, AJ, 111, 696 
Kotilainen, J. K., Ward, M. J., Boisson, C., DePoy, D. L., Bryant, L. R., \& Smith, M. G. 1992, MNRAS, 256, 125

MacKenty, J. W. 1990, ApJS, 72, 231

Maiolino, R., Ruiz, M., Rieke, G. H., \& Keller, L. D. 1995, ApJ, 446, 561

Maoz, D., Barth, A. J., Sternberg, A., Filippenko, A. V., Ho, L. C., Macchetto, F. D., Rix, H. -W., \& Schneider, D. P. 1996, AJ, 111, 2248

Mauder, W., Weigelt, G., Appenzeller, I., \& Wagner, S. J. 1994, A \& A, 285, 44

Mihos, C. J., \& Hernquist, L. 1994a, ApJ, 425, L13

Mihos, C. J., \& Hernquist, L. 1994b, ApJ, 431, L9

Miles, J. W., Houck, J. R., \& Hayward, T. L. 1994, ApJ, 425, L37

Moles, M., Márquez, I., \& Pérez, E. 1995, ApJ, 438, 604

Mouri, H., \& Taniguchi, Y. 1992, ApJ, 386, 68

Noguchi, M. 1988, A \& A, 203, 259

Norman, C. A., \& Scoville, N. Z. 1988, ApJ, 332, 124

Oliva, E., Origlia, L., Kotilainen, J. K., \& Moorwood, A. F. M. 1995, A \& A, 301, 55

Ostriker, J. P., \& Tremaine, S. 1975, ApJ, 202, L113

Pier, E. A., \& Krolik, J. 1993, ApJ, 418, 673

Pier, E. A., Antonucci, R., Hunt, T., Kriss, G., \& Krolik, J. 1994, ApJ, 428, 124

Pogge, R. W. 1989, ApJ, 345, 730

Quinn, P. J., Hernquist, L., \& Fullagar, D. P. 1993, ApJ, 403, 74

Poynting, J. H. 1903, Phil. Trans. Roy. Soc. London, Ser. A., 202, 525

Rafanelli, P., Violato, M., \& Baruffolo, A. 1995, AJ, 109, 1546

Rees, M. J. 1984, ARA \& A, 22, 471

Rieke, G. H. 1992, in Relationships Between Active Galactic Nuclei and Starburst Galaxies, ed. A. V. Filippenko (ASP Conf. Ser. 31, San Francisco 1992), 61

Robertson, H. P. 1937, MNRAS, 97, 423

Rodríguez-Espinosa, J. M., Rudy, R. J., \& Jones, B. 1987, ApJ, 312, 555

Sanders, D. B., et al. 1988, ApJ, 325, 74

Schmitt, H. R., Kinney, A. L., Storchi-Bergmann, T., \& Antonucci, R. 1997, ApJ, 477, 623 
Scoville, N. Z. 1992, in Relationships Between Active Galactic Nuclei and Starburst Galaxies, ed. A. V. Filippenko (ASP Conf. Ser. 31, San Francisco 1992), 159

Scoville, N. Z., Sargent, A. I., Sanders, D. B., \& Soifer, B. T. 1991, ApJ, 366, L5

Shlosman, I., Begelman, M. C., \& Frank, J. 1990, Nature, 345, 679

Simkin, S. M., Su, H. J., \& Schwarz, M. P. 1980, ApJ, 237, 404

Storchi-Bergmann, T., Baldwin, J. A., \& Wilson, A. S. 1993, ApJ, L11

Storchi-Bergmann, T., Eracleous, M., Livio, M., Wilson, A. S., Filippenko, A. V., \& Halpern, J. P. 1995, ApJ, 443, 617

Storchi-Bergmann, T., Rodríguez-Ardila, A., Schmitt, H. R., Wilson, A. S., \& Baldwin, J. A. 1996b, ApJ, 472, 83

Storchi-Bergmann, T., Wilson, A. S., \& Baldwin, J. A. 1996a, ApJ, 460, 252

Taniguchi, Y. 1987, ApJ, 317, L57

Taniguchi, Y. 1992, in Relationships Between Active Galactic Nuclei and Starburst Galaxies, ed. A. V. Filippenko (ASP Conf. Ser. 31, San Francisco 1992), 357

Taniguchi, Y., \& Mouri, H. 1992, in Relationships Between Active Galactic Nuclei and Starburst Galaxies, ed. A. V. Filippenko (ASP Conf. Ser. 31, San Francisco 1992), 365

Taniguchi, Y., Murayama, T., Nakai, N., Suzuki, M., \& Kamaya, O. 1994, AJ, 108, 468

Taniguchi, Y., Sato, Y., Kawara, K., Murayama, T., \& Mouri, H. 1997, A \& A, 318, L1

Taniguchi, Y., \& Wada, K. 1996, ApJ, 469, 581

Telesco, C. M., Becklin, E. E., Wynn-Williams, C. G., \& Harper, D. A. 1984, ApJ, 282, 427

Terlevich, R., \& Melnick, J. 1985, MNRAS, 213, 841

Terlevich, R., Tenorio-Tagle, G., Franco, J., \& Melnick, J. 1992, MNRAS, 255, 713

Tremaine, S. 1981, in The Structure and Evolution of Normal Galaxies, ed. S. M. Fall, \& D. Lynden-Bell (Cambridge: Cambridge University Press), 67

Umemura, M., Fukue, J., \& Mineshige, S. 1997a, ApJ, 479, L97 (UFM97)

Umemura, M., Fukue, J., \& Mineshige, S. 1997b, ApJ, submitted (UFM97)

Ward, M. J., Martin, E., Fabbiano, G., Carleton, N. P., Willner, S. P., \& Lawrence, A. 1987, ApJ, 315, 74

Weedman, D. W. 1983, ApJ, 266, 479

Weigelt, G. et al. 1991, ApJ, 378, L21 
Wilson, A. S. 1988, A \& A, 206, 41

Wilson, A. S., Baldwin, J. A., Sun, S. D., \& Wright, A. E. 1986, ApJ, 310, 121

Wilson, A. S., Helfer, T. T., Haniff, C. A., \& Ward, M. J. 1991, ApJ, 381, 79

Zaritsky, D., Smith, R., Frenk, C., \& White, S. D. M. 1997, ApJ, 478, 39 